\title{
High-Resolution Definition of Subsurface Heterogeneity for Understanding the Biodynamics of Natural Field Systems: Advancing the Ability for Scaling to Field Conditions
}

\author{
Principal Investigator: \\ E.L. Majer, Tel: 510/495-6709, Fax: 510/486-5686, email: ELMajer@lbl.gov \\ Lawrence Berkeley National Laboratory \\ Mail stop 90-1116
}

Co-Investigator: F. J. Brockman (PNNL)

\subsection{TASK DESCRIPTION}

This research is an integrated physical (geophysical and hydrologic) and microbial study using innovative geophysical imaging and microbial characterization methods to identify key scales of physical heterogeneities that affect the biodynamics of natural subsurface environments. Data from controlled laboratory and in situ experiments at the INEEL Test Area North (TAN) site are being used to determine the dominant physical characteristics (lithologic, structural, and hydrologic) that can be imaged in situ and correlated with microbial properties. Emphasis is being placed on identifying fundamental scales of variation of physical parameters that control transport behavior relative to predicting subsurface microbial dynamics. The outcome will be an improved understanding of the relationship between physical and microbial heterogeneity, thus facilitating the design of bioremediation strategies in similar environments. This work is an extension of basic research on natural heterogeneity first initiated within the DOE/OHER Subsurface Science Program (SSP) and is intended to be one of the building blocks of an integrated and collaborative approach with an INEEL/PNNL effort aimed at understanding the effect of physical heterogeneity on transport properties and biodynamics in natural systems. The work is closely integrated with other EMSP projects at INEEL (Rick Colwell et al.) and PNNL (Fred Brockman and Jim Fredrickson).

\subsection{OBJective}

The overall goal of this research is to contribute to the understanding of the interrelationships between transport properties and spatially varying physical, chemical, and microbiological heterogeneity. The research is being carried out in both the laboratory and at DOE field sites under natural conditions. This work addresses issues that will aid in the understanding of what scales one must sample in order to design effective remediation strategies. A critical question is the existence of one or more self-averaging quantities for microbial properties. Stated in another fashion: Are the microbial properties a function of the "randomness" of the media? If so, what is the size of the representative volume at which the significant properties can be characterized? A specific goal of the research is to advance the understanding of how to effectively use the information from geophysical imaging (i.e., volumetric measurements of physical properties) to predict the effect of physical heterogeneity and fluid transport properties on microbial behavior. A key hypothesis being addressed is that nutrient flux and transport properties are key factors in controlling microbial dynamics. A related hypothesis is that complexity of the subsurface environment is also correlated to the behavior of microorganisms.

Geophysics is ideally suited for extrapolating measurements made in a borehole to the large-scale volume away from the hole. High resolution seismic measurements are being used to resolve structure at the sub-meter scale. With this information on location of fractures, layer continuity, layer thickness and other structural and lithologic features, improved predictive models on flow 
and transport can be developed and applied. This is then used to correlate to the microbial behavior and distribution. In this application, geophysical measurements are made between holes to assess the continuity and homogeneity of the intervening material at the TAN site. Seismic imaging is being done in the fractured basalts at the TAN site using advanced processing and multicomponent imaging. Specifically, several different processing approaches are being used to obtain improved information on physical properties controlling transport behavior, for example;

- Conventional and advanced ray and waveform tomography

- Using guided/channel waves

- Using scattered energy from voids/high contrast anomalies

- Crosswell reflection imaging

\subsection{Progress to Date of the Geophysical Imaging}

During 1997 the geophysics concentrated on obtaining crosswell seismic data at the sub-meter resolution scale at several wells in the TAN area. Tomographic imaging was carried out for crosswell pairs, T-GRW to TAN-9, TAN-25 to T-GRW, TAN-25 to TAN-26, and TAN-25 to TSF-05. Inversions were produced for the T-GRW to TAN-9 and TAN-25 to T-GRW, TAN-25 to TAN-26, and TAN-25 to TSF-05 well pairs. An example is the T-GRW/TAN-9 well pair, which produces the most complete velocity tomogram (Figure 1). The ray coverage was good from 64 to $94 \mathrm{~m}$, and the inversion shows little effect of any deviations of the wells. The tomographic results show three low-velocity zones: one about $2 \mathrm{~m}$ thick between depths of 66 and $68 \mathrm{~m}$, a thicker one at a depth of 74 to $78 \mathrm{~m}$, and one at a depth of 85 to $87 \mathrm{~m}$ which corresponds to the low-velocity zone seen in the TAN-25/T-GRW inversion. The middle low-velocity zone is the thickest and contains the lowest velocities, well below $3000 \mathrm{~m} / \mathrm{s}$. The lower low-velocity zone is not well defined here.

From the seismic imaging several results are evident. The geology of the surveyed area is quite varied, with large changes in seismic velocity and attenuation with depth. From our experience in other fractured environments, this indicates extreme variability in fracture/rock properties and from past experience is an indication of fracture permeability. In all well pairs the tomography has given good resolution on structure and possible areas of "fast paths." Although there is strong evidence that the low-velocity zones imaged with the tomography are more permeable, further analysis and correlation with other data (well logs, tracer data, etc.) and refinement using corrected hole locations from deviation surveys should yield more definitive results on the actual identification of permeable pathways and structure controlling transport.

\subsection{Microbial Characterization and Scale Effects}

The objective of this work is to characterize small-scale variations in subsurface microbiological properties for the following purposes: providing quantitative estimates (using variogram models) of the spatial variability of these properties; and understanding the relationships between microbiological, physical, and chemical properties (using cross-correlation models). These activities will help identify to what extent geophysical and geochemical properties control the spatial variability of microbiological properties. An additional objective is to determine how the use of different sample supports affects the measurement of microbiological properties. The definition of an appropriate averaging scale is critical in providing more accurate input into predictive models of contaminant transport and will improve the precision of the simulations. The final objective is to quantify changes in the spatial structure and magnitude of microbiological properties following the impingement of a contaminant plume. An associated objective is to determine how the contamination event affects the measurement of microbiological properties at different sample supports. 


\subsection{Progress to Date of the Microbial Work}

\subsection{TAN-33}

Rock samples were obtained from one EMSP-funded borehole in FY97. Although we worked closely with personnel at INEEL, it was not possible (given their funding, schedule, and logistical considerations) to obtain the number, type, and quality of rock samples required for meeting the stated objectives. However, in FY98 we will be able to address our first two objectives based on sampling and analysis of groundwater from the borehole described above. In TAN-33, after a delay in obtaining samples from the EMSP-funded borehole, we obtained samples from a Subsurface Science Program-funded borehole to refine DNA extraction protocols in basaltic rock material. The TAN-33 borehole was located in a region containing low levels of contamination (less than $0.5 \mathrm{ppm}$ trichloroethylene, TCE). Community DNA was successfully extracted from several of the rock samples, the $16 \mathrm{~S}$ rRNA genes were amplified and cloned, and phylogenetic analysis was performed.

\subsection{TAN-37}

Compared to TAN-33, the TAN-37 borehole is closer to the source of contamination and contained 5-10 times more TCE. Microbial activity (determined by other investigators) in the TAN-37 borehole was much higher than in the TAN-33 borehole. DNA was extracted and successfully amplified from all rock samples to date; these samples correspond to the depths where the multilevel groundwater sampling will occur in FY98. DNA extraction is being performed on the remaining 15 rock samples from the TAN-37 borehole. Timecourse degradation (1- 12 weeks) of 14C-labeled organic substrates (methane and phenol aerobically; lactate and methanol anaerobically) and 14C-trichloroethylene, in the presence and absence of additional inorganic and organic nutrients, was performed on 8 rock samples, and analysis by gas chromatography-gas proportional counting is underway. 


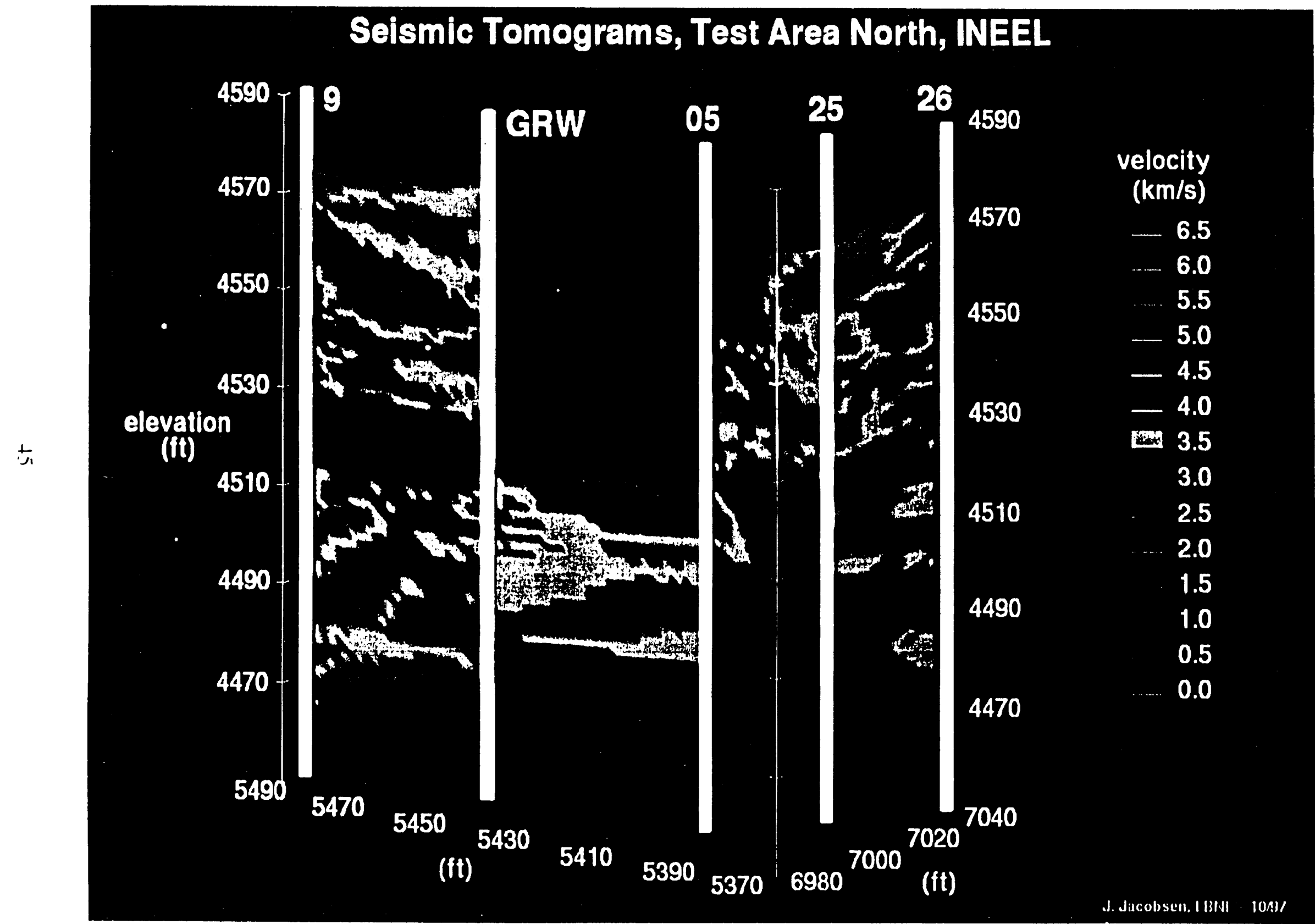

Figure 1. T-GRW/TAN-9 well pair. 